\title{
BIOSCIENCE LEARNING IN CLINICAL PLACEMENT: THE EXPERIENCES OF PRE- REGISTRATION NURSING STUDENTS
}

\section{ABSTRACT}

Aims and objectives. The aim of this study was to explore student nurses' views and experiences of bioscience learning in clinical placement. The study focussed on (1) how relevant students perceive bioscience knowledge to their professional role; (2) what opportunities students have to apply bioscience knowledge during clinical placements; (3) what students perceive about the support they receive from placement mentors regarding bioscience learning.

Background. Bioscience knowledge is required for safe and effective practice but is an area that students find challenging. Clinical placements offer students the ideal environment to integrate bioscience into clinical decision making. There is however a lack of research addressing specifically students' placement learning of bioscience.

Design. An explanatory sequential mixed methods approach was adopted.

Methods. The research involved two phases. In phase one predominantly quantitative data was collected via a survey. 112 final year BSc nursing students from across fields completed the survey (response rate $=66 \%$ ). The results of this phase were then built upon by conducting three focus groups $(n=17)$ in a second qualitative research phase.

Results. Whilst students acknowledged the relevance of bioscience to their nursing role, this study suggests that its importance is not recognised as widely by practice educators. Findings highlight inconsistencies in the quality of mentor support, the opportunities for students to learn and the priority that bioscience is given in placement education.

Conclusions. This study demonstrates the need for more explicit bioscience criteria in placement assessments and a greater level of mentor education to ensure more consistency in the standard of placement learning of bioscience. 
Relevance to clinical practice. Providing insight into factors that influence student learning of bioscience during clinical placements provides focus on how HEls and practice educators can better support students to confidently apply bioscience knowledge in order to deliver safe and effective patient care.

Keywords: Bioscience, clinical placements, nurse education, placement learning, preregistration, patient care, clinical decision making.

What does this paper contribute to the wider global clinical community?

This paper

- $\quad$ Provides insight into factors that influence nursing students' learning of bioscience during clinical placements.

- $\quad$ Provides direction for how HEls and practice educators can enhance the quality and provision of bioscience education in practice settings. 


\section{INTRODUCTION}

A working knowledge of bioscience underpins many areas of nursing practice. There has been much deliberation however as to whether nurse education is adequately equipping students with the bioscience knowledge they require for safe and effective practice (Jordan \& Reid 1997, Prowse \& Heath 2004, McVicar et al. 2010). It is well documented that student nurses find science to be one of the most challenging areas of their course and they can struggle with both understanding and applying bioscience knowledge (Caon \& Treagust 1993, Craft et al. 2013). This on-going difficulty to grasp and apply key scientific principles appears to transfer into clinical practice, as registered nurses report being unable to draw on a sound bioscience knowledge base to inform their clinical reasoning (Clancy et al. 2000, McVicar et al. 2010, Davies 2010, Logan \& Angel 2011).

Whilst studies have focused on exploring students' overall experiences of bioscience learning in nursing programmes (Courtney 1991, Clancy et al. 2000, Davies 2010) or addressed innovative ways in which students can be better supported in their learning of bioscience (McVicar et al. 2014), there is little research specifically addressing placement learning of bioscience (McVicar et al. 2010, Logan \& Angel 2011). Furthermore, these studies have investigated placement learning from a mentor / registered nurse perspective rather than exploring students' own perceptions. This is surprising as the clinical arena is arguably the best environment to apply and integrate bioscience knowledge to practice. In the United Kingdom (UK), learning and assessment in practice constitutes a significant proportion of preregistration programme time with $50 \%$ of nursing students' learning occurring on placement. Placement learning therefore makes a substantial contribution to the learning experience but appears to be an area that has been largely overlooked in the literature regarding bioscience education in healthcare. 
This paper is based on research undertaken as part of a wider study to explore the placement experiences of students from different health professions. The study seeks to investigate the support and opportunities available for pre-registration healthcare students to learn and apply bioscience within the clinical setting. The findings reported here concern student nurses' experiences of bioscience learning during clinical placements.

\section{BACKGROUND}

The biosciences that provide theoretical underpinning to nursing practice are defined by European directive 2005/36/EC as including anatomy, physiology, pathology, microbiology, biophysics, biochemistry, radiology, pharmacology and dietetics (Nursing and Midwifery Council (NMC) 2010). Smales (2010) extends this definition by also including genetics and immunology. Internationally, their place in nurse education and the tensions between science and nursing, often referred to as 'the bioscience problem' (McVicar, 2009), have been a matter of continuing debate.

A major concern is that there has been a gradual devaluing of bioscience within the nursing profession, as nursing has moved away from a medical model of care (Clarke 1995, Wynne et al. 1997, Larcombe \& Dick 2003). It has been suggested that a shift of emphasis towards the behavioural and social sciences has resulted in the biosciences being marginalised within both nursing practice and the nursing curricula (Wynne et al. 1997, Jordan 1999, Logan \& Angel 2011, Taylor et al. 2015). This debate has been on-going for decades. Issues highlighted with regards the inclusion and delivery of bioscience in nursing programmes date back nearly forty years (Wilson 1975). Concerns raised then regarding an unstructured curriculum, lack of a national syllabus and limited time in an over-crowded curriculum are still being voiced today (Smales 2010, Taylor et al. 2015).

Certainly student feedback, evidenced by university wide student satisfaction surveys, course evaluations and research studies, consistently indicate that students perceive the amount of bioscience content in their curriculum to be inadequate and that there is a need for greater 
application and more active forms of teaching (Fell \& James 2012; Taylor et al. 2015). Whilst educators have reported on a range of learning and teaching strategies aimed at improving student learning of bioscience, a recent literature review of such curriculum innovations over the past twenty years indicates that evidence on the impact of these innovations on knowledge acquisition is limited (McVicar et al. 2014).

To-date, focus has been mainly directed at improving university delivered teaching or on-line resources. Yet students are reported to learn bioscience most effectively when knowledge is related to clinical practice (Davies et al. 2000, Prowse 2003, Davies 2010). Clinical placements should, therefore, afford students the ideal environment to link bioscience theory to practice. It has been suggested, however, that the quality of placement learning may be hindered by mentors having poor understanding or confidence in their bioscience knowledge (McVicar et al. 2010, Logan \& Angel 2011). These findings warrant further investigation to provide greater insight into factors that influence nursing students' placement learning of bioscience.

This study develops the evidence on nursing students' learning of bioscience beyond the classroom by specifically considering the learning that occurs during clinical placements. Three research questions are addressed:

1. How relevant do students perceive bioscience knowledge to their professional role?

2. What opportunities do students have to apply bioscience knowledge to practice during clinical placements?

3. What do students perceive about the support they receive from their placement mentors regarding their bioscience learning?

\section{METHODS}

\section{Design}


The study was conducted in one higher education institution (HEI) in the 2013/2014 academic year. An explanatory sequential mixed methods approach was used involving two phases. In phase one, predominantly quantitative data was collected via a survey. The results of this stage of the project informed the design of focus group interviews which were conducted as part of a second qualitative research phase. In such an approach qualitative data is used to further explain and expand upon the quantitative data gathered (Creswell, 2014).

\section{Research instruments}

Phase one: survey

The survey used largely closed questions in relation to exploring each of the three research questions. Survey items relevant to the findings reported in this paper are presented in Tables 3 and 4 . In addition, the survey included three open questions so that respondents could qualify ratings that they had given concerning both bioscience relevance and mentor support. Together with the quantitative data, these open comments helped the researchers to identify specific areas to address further in focus groups within the qualitative phase of the project. The survey questions were written by the research team and piloted on a small student sample prior to administration for face validity and clarity.

Phase two: focus group interviews

As part of the mixed methods design, focus groups were conducted to elaborate on the data gained from the survey. Following preliminary analysis of phase one, a question schedule was developed by the research team to further explain and add depth to the survey findings. Fig. 1 outlines the key topics addressed within the schedule. Each focus group was conducted on campus by the lead project researcher, who was unknown to the students, and audio recorded with the consent of all participants. Another project researcher transcribed the sessions verbatim.

[insert Figure 1 here] 
To maximise the validity of the research instruments, items asked within the survey and focus groups were all mapped against the three overarching project research questions. This process also ensured that triangulation of data could occur between both phases of the project.

\section{Sample}

Project participants comprised final year BSc Nursing students from the Adult, Child and Mental Health fields. Final year students were selected as they would have completed a range of placements prior to involvement in the study and, as a result, have various experiences to draw upon. Inclusion of all three nursing fields further enhanced the validity of the study as data could be triangulated across the groups. Due to cohort sizes, the Adult field students were separated into three groups. The study was conducted with one group from this field, and the entire cohort of the Child and Mental Health fields.

\section{Data collection process}

In phase one, paper-based surveys were distributed either at the beginning or end of campusbased teaching sessions and respondents were able to complete and return them in the session or complete them in their own time and return to a collection box in a specified location. Students were also asked to consider participating in follow-up focus groups. Following phase one, potential focus group participants were contacted via email and field-specific focus groups arranged. From the cohort of Mental Health students, none wished to participate in a focus group. Consequently, the second intake group for the 2013/2014 academic year were approached and eight students agreed to participate. This was the only focus group where participants had not previously been within the sample invited to complete the survey. Whilst this deviation from the original survey sample could not be avoided, it did offer further opportunities for triangulation of the survey data gained.

\section{Data analysis}


Quantitative analysis

Following the mixed methods design of this study, the quantitative data was analysed prior to the second phase of the project. The Statistical Package for Social Sciences (SPSS version 21) was utilised for descriptive statistics (primarily overall frequencies and frequencies for field sub-groups) and inferential statistical analysis. As the data was ordinal level (derived from likert scales), a series of non-parametric Kruskal-Wallis tests were performed to determine the statistical significance of differences between field responses. The level of statistical significance was set at 0.05 (two-tailed). For post hoc analysis, a series of Mann Whitney $U$ tests were undertaken for those variables demonstrating statistical significance. A reduced significance of alpha $=0.017(0.05 / 3)$ was applied as a Bonferroni correction, to take account of Type 1 errors (Pallant, 2013).

Qualitative analysis

The comments from the survey open questions were preliminarily coded to identify the most prominent themes arising and further analysed with the qualitative data in phase two. Once phase two was complete, all qualitative data was interrogated to 'establish analytical categories that address the research questions' (Arskey and Knight, 1999, p.162). In-depth analysis occurred by coding data across the three nursing fields to identify emerging patterns and themes. To ensure reliability and consistency in this process, the research team (lead and researcher) each independently read the transcripts and then agreed upon categories and themes.

\section{Ethical considerations}

Ethical approval was obtained from the University Faculty of Health Research Committee. All participants gave their consent prior to taking part. They were assured of the confidentiality and anonymity of their data, and of their rights as research subjects. 


\section{RESULTS}

\section{Demographics}

Survey

From a total of 169 students, 112 responses were received (66\% response rate). Respondents who completed the survey self-identified into the following nurse cohort groups: Adult 38\%; Child 33\%; Mental Health 29\%. Students' background knowledge of bioscience on joining the course varied widely, with entry qualifications ranging from one student having no biological science qualification at all (1\%) to some with degrees in the subject area (9\%) (Table1).

[insert Table 1 here]

Focus groups

Seventeen students took part in three field specific focus groups. Participant characteristics are shown in Table 2.

[insert Table 2 here]

\section{Findings}

The results present the main quantitative and qualitative findings in relation to the three research questions. Data are connected to show particularly how the qualitative results expand upon the quantitative survey findings (Zhang \& Creswell, 2013).

\section{Relevance of bioscience to nursing profession}

\section{Quantitative results}

In the survey, students were asked to rate how important they believed knowledge of bioscience is to their professional role. Data demonstrated that students from all fields clearly appreciated the need for bioscience knowledge in nursing practice, as the majority of the overall sample $(97 \%)$ rated it as 'essential', 'very important' or 'important' (Table 3). 
There was a significant difference in ratings across fields $(p<0.0005)$. Post hoc tests revealed that Mental Health students attached less importance of this knowledge to their role compared to Child and Adult student nurses ( $p<0.0005$ in both cases).

[insert Table 3 here]

\section{Qualitative findings}

In an open comment section of the survey, students were asked to explain the reasons for their ratings. Many students from all fields expressed the belief that bioscience is central to day to day practice as a nurse. To elaborate on the survey comments, focus group participants were asked to explain further their views about the importance of bioscience. Consistent with the survey results, all focus group participants emphasised the importance of bioscience to their professional roles. Three core themes emerged from the data, illuminating students' perspectives about the relevance of bioscience knowledge.

Theme one: Foundational knowledge for competence

Students overwhelmingly indicated that bioscience provides fundamental underpinning knowledge (foundational knowledge) required for nurses to act competently in their role. The link between underpinning bioscience knowledge and understanding conditions, observations and treatment choices was frequently emphasised by students:

I think it's important...to have an underpinning knowledge of conditions because if you don't know what's wrong with a patient and you don't recognise what they've got, how can you treat it? (FG/NA6 $\left.{ }^{1}\right)$.

Such foundational knowledge was linked to justifying their actions as nurses. In addition, the increasing need for bioscience knowledge in relation to more extended and autonomous roles was also recognised, typified by the comment below: 
I think [bioscience knowledge] is important as the nursing role is developing and you've got more responsibilities (FG/NA3).

Theme two: Foundational knowledge for patient care

Also identified was the link between bioscience knowledge and patient care. Students from all fields explicitly referred to the importance of bioscience knowledge for supporting patient care and the link between bioscience knowledge and the safety of the patient was highlighted:

If there isn't much knowledge on this then I feel patients may be at risk (S/NMH25).

Bioscience knowledge was also viewed as enabling students to better interact and communicate with their patients. During focus group discussions, however, some participants expressed concern that patients expected them to have a far greater knowledge of bioscience than they actually had:

and it can be quite embarrassing when you've got to say to a family 'I don’t know

in that much detail' because they want to know, they want to know everything... because they see you as the expert (FG/NMH2).

\section{Theme three: Foundational knowledge for confidence}

Comments suggest that the acquisition of bioscience knowledge increases students' confidence to undertake their role and challenge aspects of practice if necessary. Students indicated that bioscience knowledge helps them to feel confident as health professionals and further gives '...patients the confidence that you know what you're doing' (FG/NC3). One participant stated specifically that having this type of knowledge would give them 'a good grounding' (FG/NMH7) to challenge consultants or other health professionals if they had concerns about certain aspects of their patient's care. In addition, students across fields commented that an underpinning knowledge of bioscience helped them to communicate more effectively with colleagues: 
I feel that a professional nurse should understand the physiological effect of drugs/illness/injury on a patient and be able to...hold appropriate clinical conversations with colleagues (S/NA36).

\section{Opportunities to apply bioscience knowledge to practice}

\section{Quantitative results}

The survey explored the degree to which students felt that they had the opportunity to apply their bioscience knowledge to their placement activities. Firstly, students were asked about the extent to which the importance of applying bioscience was emphasised during their placements. The majority of the overall sample (53\%) indicated that the importance of applying bioscience was emphasised 'to some extent', suggesting moderate emphasis has been placed on bioscience in many cases, with $32 \%$ of respondents reporting that it had been emphasised to 'a great extent'. 15\% of students however reported less positive experiences where bioscience had been emphasised to a little or no extent. This trend remains across field specific data (Table 3). Discussions with mentors were identified as a main method of learning bioscience on placement (Table 4). When asked in the survey how often they discussed with their mentors the science relating to patient care, opportunities appeared variable across the sample. Whilst just over half (55\%) reported 'often' or 'very often', a high proportion (41\%) indicated that these discussions occurred 'not very often', and 4\% said that they 'never' happened. A Kruskal-Wallis test revealed a significant difference across fields $(p=0.04)$. Post hoc tests highlighted that Mental Health students discussed patient science with their mentor less often than Adult students $(p=0.016)$. Nearly all survey respondents $(97 \%)$ indicated that they considered discussions about the science relating to patient care relevant to their placement experiences (Table 3).

[Insert Table 4 here]

\section{Qualitative findings}


To further explore factors which contribute to the variability in opportunities and support evidenced in the survey, students were asked within focus group interviews to think about when and how they may focus on bioscience within a typical day on placement. Three particular themes emerged as factors which influence placement learning opportunities of bioscience: placement attitudes, documentation and time.

\section{Theme one: placement attitudes}

Opportunities for application of knowledge appeared to be limited to some extent by placement attitudes to bioscience. Concerns were raised that a 'negative ward culture' (S/NMH7) sometimes meant that bioscience was not a priority within placement settings. It became apparent in focus group discussions that the extent to which bioscience was emphasised was often dependent upon the stage of the students' course and the placement area. Many Adult field participants suggested that the biosciences are not emphasised to a great extent during their first and second year placements. Instead, there is a greater focus on completing the more routine and mundane practical tasks, such as 'washing [and] toileting patients' (FG/NA2). It is within their final year placements that students in this group indicated that the biosciences are more explicitly emphasised. For some, this situation leads to their final year placements feeling 'like a massive jump from barely anything to everything' (FG/NA4).

Various Adult and Child field participants reported that bioscience knowledge is often emphasised more in specialist placement areas, such as critical care, which subsequently provide greater opportunities to apply this knowledge:

In the more specialised area you would unconsciously always be using bioscience... (FG/NC2).

Theme two: documentation

Students also suggested that a lack of explicit bioscience content in placement documentation meant that there was little incentive to prioritise such learning: 
no requirement in placement documents for mentors to assess biological knowledge in depth (S/NA16).

This was emphasised by one focus group participant who regarded the placement document as potentially focusing mentors' attention 'on the tick boxes in the document' (FG/NC2) rather than on bioscience-related aspects such as anatomy and physiology.

Theme three: time

Students indicated that they have limited time to discuss bioscience with their mentors whilst on placement. Reasons reported were staff shortages, high patient case loads and busy wards. The value students placed, however, on being able to take the time and sit down with a mentor to review aspects of bioscience was clearly evident as several students identified 'having more time with mentors' (S/NMH17) and 'time to talk' (S/NC2) as being important factors that supported their learning.

Significantly it was evident that opportunities to learn bioscience were very much dependent upon mentor support. This theme is explored in relation to the final research question.

\section{Mentor support}

\section{Quantitative results}

Within the survey, students were asked to rate the bioscience knowledge of their mentors and the overall support provided by their mentors with regards their bioscience learning on placement. Overall, $79 \%$ of students rated their mentors' bioscience knowledge to be 'good', 'very good' or 'excellent' and the majority of students (66\%) gave a favourable opinion as to the support they received (Table 3). The data does highlight that there is still a substantial percentage of students that perceived the support they received as substandard, with $21 \%$ of students overall rating their mentors' knowledge base to be only 'fair' or 'poor' and a third reporting the bioscience support they received from mentors as less than 'good'. A significant variation was evident across fields $(p=0.02)$ in relation to perceived support. Post hoc tests 
showed that Adult field students have a more positive perception of bioscience support provided by mentors than Mental Health students $(p=0.008)$.

\section{Qualitative findings}

Students were asked within the survey to comment on factors that aided and hindered working with their mentors. Mentor support was explored further in focus group interviews where students were asked about the extent to which they had learnt from their mentor in relation to underpinning bioscience and whether mentors had been proactive in supporting bioscience learning. Evident particularly from focus group discussions (all fields) was that mentor support can be very variable. Students reported a range of experiences with some able to describe extremely positive learning experiences with proactive, knowledgeable mentors. Many, however, indicated receiving very little explicit bioscience teaching. When considering factors that may account for such perceived variability, three core themes emerged: mentor attitudes, mentor knowledge and mentor enthusiasm and ability to teach.

\section{Theme one: Mentor attitudes}

Reference was made again to a culture where bioscience is not considered a priority and as such is not judged to be an integral part of a nurse's role. Students perceived such views as having a significant impact on the support provided by some mentors:

I've had a lot of different experiences of mentors and some seem to be very geared up towards talking about bioscience and some almost completely disregard it as not being part of what a nurse does... (FG/NMH8)

\section{Theme two: Mentor knowledge}

Students repeatedly identified mentors' knowledge to be an important factor in determining the support they received about biosciences. Mentors with inadequate or insufficient bioscience knowledge were viewed by students to be a hindrance to good support: 
Mentor knowledge has to be good (S/ NC18).

Concerns were raised about the extent of mentors' bioscience related knowledge. Various students from all fields suggested that the knowledge and understanding of some mentors was 'lacking' (S/NC16) which was considered 'quite scary' (FG/NMH7) and even 'terrifying' (FG/NMH6). Commonly, students appeared to perceive a lack of consistency regarding the nature of mentors' bioscience knowledge:

it's very variable between mentors [as] to what knowledge they've got (FG/NA6).

Theme three: Mentors' enthusiasm and ability to teach

Whilst many students identified mentors' bioscience knowledge as an important factor in determining the support that they received, some also indicated that mentors having the relevant knowledge did not always make them effective in supporting their bioscience learning. Qualitative comments suggest that participants perceive 'good' mentors as those who 'push' (FG/NA6) students in bioscience learning by asking them questions and testing their knowledge. Less effective mentors were viewed by students as those who appeared to brush off students' bioscience-related questions and ignore relevant opportunities to test their mentees' knowledge. Significantly, participants appeared to associate a lack of bioscience knowledge with reluctance in mentors to engage in teaching and learning activities:

I've had a few mentors where they've been 'go find it out for yourself and then come and tell me', but a lot of that is because they don't know themselves and [it's] getting you to do the leg work (FG/NA4).

However, there was acknowledgement that responsibility for enhancing bioscience learning during placement rested with students themselves as well as their mentors. As a result, students in each field recognised the need to be proactive in seeking out relevant learning opportunities. This proactiveness was also viewed as important when working with mentors who appeared to be less than competent or enthusiastic in their role as 'teachers'. Various 
participants suggested that not all mentors seemed 'inspir[ed]...to teach' (FG/NMH8) or even simply 'willing to teach' (FG/NA5), which was acknowledged to have adverse effects on the extent of their learning.

Such findings may explain why students identified that their learning occurs on a wider scale than just with their mentors. Students acknowledged the value of spending time in other departments and tapping the 'wealth of knowledge and experience' (FG/NMH2) from other health care professionals. Patients were also referred to as being a valuable source of knowledge:

the best person to ask sometimes is the patient because they can tell you all about it, they can probably tell you more than what your mentors can, so that's been really useful on quite a lot of placements (FG/NA4).

\section{DISCUSSION;}

As reported previously, the influence of bioscience knowledge to nursing competence and patient care has been widely recognised in the literature (Wynne et al. 1997, Jordan \& Reid 1997, Prowse \& Heath 2004, Danielson \& Berntsson 2007). Yet the value that is placed on bioscience knowledge within the profession continues to be a matter for some debate. Certain studies have demonstrated that both registered staff (Clancy et al. 2000, Davies 2010) and nursing students (Caon \& Treagust 1993, Thornton 1997, Clancy et al. 2000, Friedel \& Treagust 2005) do appear to recognise the need for sound bioscience knowledge, although it has been suggested that there has been a gradual devaluing of the use of such knowledge in both clinical practice and placement education (Logan \& Angel, 2011).

Our research clearly supports the opinion that students appreciate the relevance of bioscience knowledge to nursing, as almost all students considered it to be either essential or important to their profession. Respondents from all fields highlighted the need for bioscience for fundamental aspects of nursing care such as understanding a patient's condition, 
observations and treatment choices. Students notoriously find bioscience to be a challenging topic (Nicoll \& Butler 1996, Craft et al. 2013) so these findings are encouraging as it has been proposed that the key to improving nursing students' grasp of bioscience is to convince them to see the relevance of bioscience to practice (Caon \& Treagust 1993, Thornton 1997).

It is of interest that students not only recognised the underpinning nature of bioscience to understanding everyday aspects of nursing and to safe practice but also in relation to equipping them with the skills and knowledge to confidently communicate with fellow health professionals and service users. Nurses need to be 'knowledgeable doers' (Jordan 1999) who are fully informed by underpinning theory, but they also need to be 'knowledge brokers' (Logan \& Angel 2011) with an ability to share knowledge and information as part of a multi-disciplinary team and to act as patients' advocate. As Clarke (1995, p405) argued: 'Nurses should not only base their practice on sound biological knowledge but should be seen and heard to do so. This can only enhance their credibility with both patients and clients not to mention their employers'.

Certainly, it has been reported that service users expect a greater level of knowledge than nurses actually have (Jordan et al. 2000, Friedal \& Treagust 2005, Davies 2010). Our study revealed that final year students are experiencing situations where this is indeed the case and it was evident that some keenly felt their lack of bioscience knowledge and the implications that this has on their professional role.

Students highlighted the need for bioscience to be prioritised in pre-registration nurse education to ensure that they are equipped with the knowledge and understanding to undertake their roles with confidence. With the responsibilities associated with nursing currently increasing, the requirement for qualified nurses to have a better understanding of bioscience has never been more important. Davies (2010) argues however that whilst preand post-registration nurses are able to appreciate the bioscience knowledge they need, this is not necessarily well applied in clinical practice. Application of bioscience to clinical reasoning 
requires not just understanding but complex integration of knowledge to the context of patient care. Unsurprisingly, students' learning of bioscience is acknowledged to be more effective when knowledge is grounded in nursing practice (Davies et al. 2000, Prowse 2003). The clinical arena provides the perfect environment to enable students to gain experiential learning and bridge that theory-practice gap. This study however raises concerns regarding the inconsistency in the quality of support offered to students, the opportunities to learn and the priority that bioscience is given in placement education.

Whilst students overwhelmingly acknowledged the relevance of bioscience to nursing, our data suggests that its importance is not recognised as widely by mentors in placement settings, where the emphasis and opportunities to consider underpinning bioscience were seen to be variable. It appears that placement culture can have a significant influence on how bioscience is valued, and consequently the support offered to students to apply theory to practice. Where bioscience was seen as a priority, for example in specialist areas such as critical care, students were provided with more opportunities and a greater level of support. However this was not always the case and students reported placement areas where the biosciences were not emphasised to any great extent and opportunities to learn were limited by placement attitudes. Such findings echo those of Logan \& Angel (2011) who reported that a culture where the emphasis is on procedural skills can undermine theoretical underpinning and have a negative impact on student bioscience learning and application. Yet our students of today, who so clearly value bioscience knowledge, will be the mentors of tomorrow. This raises the question as to whether such cultures of practice not only limit students' opportunities to learn bioscience but can perhaps gradually erode the value placed by students and new graduates on underpinning scientific theory.

The key role that mentors play in student learning is highlighted in this study, as is the impact of mentors' own perceptions of bioscience relevance, their knowledge base and their enthusiasm and ability to teach. In recent years more emphasis has been placed on practice based learning in nursing and there is increasing awareness of the importance of effective 
mentorship to students' clinical placement experiences (Eller et al. 2014, Foster et al. 2015). Students in this study equated effective mentorship with a mentors' ability to ask questions and test their knowledge. A lack of mentors' biological knowledge was seen therefore to restrict learning opportunities to integrate theory with practice. These findings concur with the opinion expressed by McVicar et al. (2010) that the learning environment in practice is hindered by practitioners with poor bioscience understanding. Concerns regarding the level of registered nurses' bioscience knowledge and understanding have been raised for many years (Wilson 1975, Clarke 1995, Clancy et al. 2000) and more recent studies have suggested that mentors may not have adequate science background to help students apply bioscience to practice (Friedel \& Treagust 2005, McVicar et al. 2010, Davies 2010, Logan \& Angel 2011). It is therefore disappointing, but not unsurprising, that students voiced disquiet with regards variability in mentors' knowledge. There is clearly a need to develop mentors' knowledge and confidence to enable them to adequately facilitate effective student learning of bioscience. Such findings support the demand for greater emphasis being placed not only on preregistration learning but also on post-registration bioscience education.

Our findings also highlight the importance students placed on a mentor's enthusiasm and ability to teach, which are issues raised in literature considering mentorship in general (Mayall et al. 2008, Jokelainen et al. 2011). It has been suggested that effective mentors require pedagogical competence in addition to being approachable, understanding, enthusiastic, professional and confident (Eller et al. 2014, Foster et al. 2014). It is important to ensure that students are supported by practitioners that want to be mentors and have the skills and attributes to perform the role effectively. In relation to bioscience learning, many of these qualities rely not only on the scientific knowledge of mentors but also on the priority they place on bioscience within the context of competing demands and limited time. A lack of explicit bioscience content within placement documentation offers little incentive for mentors to prioritise such learning and highlights the need for practice assessments to include specific bioscience components. 
Overall, the apparent sense of students' experiences with mentors effectively being 'the luck of the draw' is unsatisfactory and suggests that there is a greater potential for variability than consistency in the extent of mentor support and opportunities for bioscience learning during clinical placements. Such findings indicate the need to incorporate clearer bioscience criteria in practice assessments and provide more systematic education, training and support to ensure that all mentors are able to actively link scientific knowledge to clinical reasoning to achieve a more consistent student experience.

A limitation of this study is that it was conducted in one higher education institution and therefore generalisability of findings cannot be assumed. Further research should explore whether the themes emerging in this study are apparent in the perceptions of nursing students in wider institutional and placement contexts. It is recommended that future studies also extend the research sample to include mentors, investigating in particular: mentors' perceptions concerning the importance and relevance of bioscience to nursing; the influence of placement culture on such perceptions; their competence and confidence in teaching bioscience to students and their views on key drivers and barriers to placement learning of bioscience.

\section{CONCLUSION}

This study demonstrates the inconsistent nature of placement provision for supporting student learning of bioscience. It suggests that the current system is failing to meet the requirements of many nursing students who clearly appreciate the need to understand and apply bioscience in order to achieve professional competence. Such findings offer evidence that professional bodies, HEls and placement providers need to work together to ensure greater consistency in the standard and quality of placement learning of bioscience, across both the UK and in countries with similar systems for supporting nursing students. Also highlighted is the need for additional mentor training and bioscience education, greater incorporation of bioscience in 
practice assessments and further research into both student and mentors' experiences of bioscience learning and teaching in clinical placement.

\section{RELEVANCE TO CLINICAL PRACTICE}

A sound knowledge of bioscience is required for safe and effective practice but is an area that students find challenging. Clinical education is a key component to learning in nurse education and offers students the ideal environment to apply and integrate bioscience into clinical decision making. This data will contribute to a greater understanding of students' experiences of placement learning of bioscience, thus providing focus on how HEls and placement providers can better support both students and mentors to ensure nurse graduates of the future are able to confidently apply bioscience to deliver safe and evidence-informed patient care.

\section{NOTES}

1. Respondent coding: $S=$ survey respondent; $F G=$ focus group participant $/ N A=$ Adult field; $\mathrm{NC}=$ Child field; $\mathrm{NMH}=$ Mental Health field.

\section{REFERENCES}

Arskey, H. and Knight, P. (1999) Interviewing for social scientists. Sage Publications Ltd, London.

Caon M \& Treagust D (1993) Why do some nursing students find their science courses difficult? Journal of Nursing Education 32, 255-259.

Clancy J, McVicar A \& Bird D (2000) getting it right? An exploration of issues relating to the biological sciences in nurse education and nursing practice. Journal of Advanced Nursing 32,1522-1532.

Clarke M (1995) Nursing and the Biological Sciences. Journal of Advanced Nursing 22, 405406. 
Courtney M (1991) A study of the teaching and learning of the biological sciences in nurse education. Journal of Advanced Nursing 16, 1110-1116.

Creswell JW (2014). Research Design: Qualitative, Quantitative and Mixed Methods Approaches, 4th Edition. Sage Publications Ltd, London.

Craft J, Hudson P, Plenderleith M, Wirihana L \& Gordon C (2013) Commencing nursing students' perceptions and anxiety of bioscience. Nurse Education Today 33, 1399-405

Davies GM (2010) What is provided and what the registered nurse needs - bioscience learning through the pre-registration curriculum. Nurse Education Today 30, 707-12.

Davies S, Murphy F, Jordan S (2000) Bioscience in the pre-registration nursing curriculum: finding the right teaching strategy. Nurse Education Today 20, 123- 135.

Danielson E \& Berntsson L (2007) Registered nurses' perceptions of preparation for professional work and development in their profession. Nurse Education Today 27, 900-908.

Eller LS, Lev EL \& Feurer A (2014) Key components of an effective mentoring relationship: A qualitative study. Nurse Education Today 34, 815-820.

Fell P \& James P (2012) Body of Evidence. Nursing Standard 27, 64.

Foster H, Ooms A \& Marks-Maran D (2015) Nursing students' expectations and experiences of mentorship. Nurse Education Today 35, 18-24.

Friedel JM \& Treagust DF (2005) Learning bioscience in nursing education: perceptions of the intended and the prescribed curriculum. Learning in Health and Social Care 4, 203-216.

Jokelainen M, Turunen H, Tossavainen K, Jamookeeah D \& Coco K (2011) A systematic review of mentoring nursing students in clinical placements. Journal of Clinical Nursing 20, 2854-2867.

Jordan S (1999) Biosciences on the margin. Nursing Standard 13, 46-48.

Jordan S \& Reid K (1997) The biological science in nursing: an empirical paper reporting on the applications of physiology to nursing care. Journal of Advanced Nursing 26, 169-179.

Jordan S, Philpin S, Davies S, Andrade M (2000) The biological sciences in mental health nursing: stakeholders' perspectives. Journal of Advanced Nursing 32, 881-891

Larcombe J \& Dick J (2003) Who is best qualified to teach bioscience to nurses. Nursing Standard 17, 38-44.

Logan PA \& Angel L (2011) Nursing as a scientific undertaking and the intersection with science studies: implications for nursing management. Journal of Nursing Management 19, 407-417.

McVicar A (2009) A post-registration solution to the bioscience problem? British Journal of Nursing 18, 149 
McVicar A, Clancy J \& Mayes N (2010) An exploratory study of the application of bioscience in practice, and its implications for pre- qualifying education. Nurse Education Today 30, 61522.

McVicar A, Andrew S \& Kemble R (2014) Biosciences within the pre-registration (pre-requisite) curriculum: An integrative literature review of curriculum interventions 1990-2012. Nurse Education Today 34, 560-568.

Mayall M, Levett-Jones T \& Lathlean J (2008) Mentorship in contemporary practice: the experiences of nursing students and practice mentors. Journal of Clinical Nursing 17,18341842.

NMC (2010) Standards for pre-registration nurse education. Nursing and Midwifery Council, London.

Nicoll L \& Butler M (1996) The study of biology as a cause of anxiety in student nurses undertaking the common foundation programme. Journal of Advanced Nursing 24, 615-624.

Pallant J (2013) SPSS Survival Manual. A Step by Step guide to data analysis using IBM $S P S S, 5^{\text {th }}$ Edition. Open University Press, Maidenhead.

Prowse MA (2003) learning and using biosciences in nursing. Part one: a review of the literature. Journal of Advanced Peri-operative Care 1, 85-93.

Prowse MA \& Heath V (2004) Working collaboratively in health care contexts: the influence of bioscientific knowledge on patient outcomes. Nurse Education Today 25,132-139.

Smales K (2010) Learning and applying biosciences to clinical practice in nursing. Nursing Standard 24, 35-39

Taylor V, Ashelford S, Fell, P and Goacher PJ (2015), Biosciences in nurse education: is the curriculum fit for practice? Lecturers' views and recommendations from across the UK. Journal of Clinical Nursing. doi: 10.1111/jocn.12880

Thornton T (1997) attitudes towards the relevance of biological, behavioural and social sciences in nursing education. Journal of Advanced Nursing 26,180-186.

Wilson KJW (1975) Study of the Biological Sciences in Relation to Nursing. Churchill Livingstone, Edinburgh.

Wynne, N, Brand S \& Smith R (1997) Incomplete holism in pre-registration nurse education: the position of the biological sciences. Journal of Advanced Nursing 26, 470-474.

Zhang W \& Creswell J (2013) The use of 'mixing' procedure of mixed methods in health services research. Medical Care 51, e51-e57. 
Focus group topics

- Importance of bioscience to professional role

- Examples of when students would use and refer to bioscience knowledge whilst on placement

- Mentor initiative in discussing bioscience

- Value of mentor support to bioscience learning

Figure 1 Topics covered within focus group discussions

Table 1: Survey participant characteristics

\begin{tabular}{llc}
\hline Characteristic & Response options & $\begin{array}{c}\text { Number of } \\
\text { respondents } \\
(\%)\end{array}$ \\
\hline \multirow{2}{*}{ Field of nursing } & Adult & $43(38)$ \\
& Child & $37(33)$ \\
& Mental Health & $32(29)$ \\
Highest Qualification in Biology & Degree & $10(9)$ \\
& A level (grade $A^{*}-$ E) or equivalent & $46(41)$ \\
& AS level or equivalent & $9(8)$ \\
& GCSE grade $A^{*}-C$ or equivalent & $45(41)$ \\
& None & $1(1)$ \\
& & \\
\hline
\end{tabular}

Table 2: Focus group participant characteristics

\begin{tabular}{lll}
\hline Field of nursing & $\begin{array}{l}\text { Number of } \\
\text { participants }\end{array}$ & Gender \\
\hline Adult & 6 & $\begin{array}{l}5 \text { female } \\
1 \text { male }\end{array}$ \\
& 3 & $\begin{array}{l}3 \text { female } \\
0 \text { male }\end{array}$ \\
Child & & $\begin{array}{l}4 \text { male } \\
4 \text { female }\end{array}$ \\
\hline
\end{tabular}


Table 3 Quantitative survey data results (total nursing sample results and results per field presented)

\begin{tabular}{|c|c|c|c|c|c|c|c|}
\hline Survey question & Rating scale & $\begin{array}{l}\text { Total } \\
\text { nursing } \\
\text { sample } \\
(\%)\end{array}$ & $\begin{array}{l}\text { Adult } \\
\text { field } \\
\text { (NA) } \\
(\%)\end{array}$ & $\begin{array}{l}\text { Child } \\
\text { field } \\
\text { (NC) } \\
(\%)\end{array}$ & $\begin{array}{l}\text { Mental } \\
\text { Health } \\
\text { field } \\
(\mathrm{NMH}) \\
(\%)\end{array}$ & $\begin{array}{l}\text { Kruskal- } \\
\text { Wallis } H \\
(p-\text { value })^{* *}\end{array}$ & $\begin{array}{l}\text { Post hoc Mann } \\
\text { Whitney U test } \\
p_{\text {-value }}^{* * *}\end{array}$ \\
\hline $\begin{array}{l}\text { In your view, how important is } \\
\text { a knowledge of bioscience to } \\
\text { your professional role? } \\
(n=111)\end{array}$ & $\begin{array}{l}\text { Unimportant } \\
\text { Slightly important } \\
\text { Important } \\
\text { Very important } \\
\text { Essential }\end{array}$ & $\begin{array}{l}0 \\
3 \\
23 \\
30 \\
44\end{array}$ & $\begin{array}{l}0 \\
2 \\
12 \\
35 \\
51\end{array}$ & $\begin{array}{l}0 \\
0 \\
22 \\
19 \\
59\end{array}$ & $\begin{array}{l}0 \\
10 \\
39 \\
35 \\
16\end{array}$ & $\begin{array}{l}16.84 \\
(<0.0005)\end{array}$ & $\begin{array}{l}\text { NA v NC } \quad 0.74 \\
\text { NA v NMH }<0.0005 \\
\text { NC v NMH }<0.0005\end{array}$ \\
\hline $\begin{array}{l}\text { Overall, to what extent was the } \\
\text { importance of applying } \\
\text { bioscience knowledge } \\
\text { emphasised during your } \\
\text { placements? } \\
(n=111)\end{array}$ & $\begin{array}{l}\text { To a great extent } \\
\text { To some extent } \\
\text { To a little extent } \\
\text { To no extent }\end{array}$ & $\begin{array}{l}32 \\
53 \\
13 \\
2\end{array}$ & $\begin{array}{l}41 \\
52 \\
5 \\
2\end{array}$ & $\begin{array}{l}27 \\
60 \\
13 \\
0\end{array}$ & $\begin{array}{l}25 \\
47 \\
25 \\
3\end{array}$ & $\begin{array}{l}5.01 \\
(0.08)\end{array}$ & - \\
\hline $\begin{array}{l}\text { Overall, how often did you } \\
\text { discuss with your mentors the } \\
\text { science related to patient } \\
\text { care? } \\
(n=109)\end{array}$ & $\begin{array}{l}\text { Very often } \\
\text { Often } \\
\text { Not very often } \\
\text { Never }\end{array}$ & $\begin{array}{l}14 \\
41 \\
41 \\
4\end{array}$ & $\begin{array}{l}19 \\
49 \\
27 \\
5\end{array}$ & $\begin{array}{l}8 \\
50 \\
42 \\
0\end{array}$ & $\begin{array}{l}13 \\
22 \\
59 \\
6\end{array}$ & $\begin{array}{l}6.62 \\
(0.04)\end{array}$ & $\begin{array}{l}\text { NA v NC } 0.263 \\
\text { NA v NMH } 0.016 \\
\text { NC v NMH } 0.084\end{array}$ \\
\hline $\begin{array}{l}\text { Do you feel that discussions } \\
\text { with your mentors about the } \\
\text { science relating to patient care } \\
\text { are relevant to your placement } \\
\text { experiences? } \\
(n=109)^{*}\end{array}$ & $\begin{array}{l}\text { Yes } \\
\text { No }\end{array}$ & $\begin{array}{l}97 \\
3\end{array}$ & $\begin{array}{l}100 \\
0\end{array}$ & $\begin{array}{l}100 \\
0\end{array}$ & $\begin{array}{l}90 \\
10\end{array}$ & - & \\
\hline $\begin{array}{l}\text { Overall, how would you rate } \\
\text { the bioscience knowledge of } \\
\text { your mentors? } \\
(n=108)\end{array}$ & $\begin{array}{l}\text { Excellent } \\
\text { Very good } \\
\text { Good } \\
\text { Fair } \\
\text { Poor }\end{array}$ & $\begin{array}{l}15 \\
28 \\
36 \\
19 \\
2\end{array}$ & $\begin{array}{l}15 \\
39 \\
29 \\
15 \\
2\end{array}$ & $\begin{array}{l}22 \\
20 \\
36 \\
22 \\
0\end{array}$ & $\begin{array}{l}6 \\
23 \\
45 \\
23 \\
3\end{array}$ & $\begin{array}{l}3.45 \\
(0.18)\end{array}$ & \\
\hline
\end{tabular}




\begin{tabular}{|c|c|c|c|c|c|c|c|}
\hline Overall, how would you rate & Excellent & 14 & 17 & 13 & 9 & & \\
\hline the support provided by your & Very good & 17 & 34 & 8 & 6 & & \\
\hline mentors regarding your & Good & 35 & 25 & 46 & 38 & & NA v NC 0.060 \\
\hline bioscience learning on & Fair & 23 & 17 & 19 & 34 & 7.86 & $\mathrm{NA} \vee \mathrm{NMH} 0.008$ \\
\hline $\begin{array}{l}\text { placement? } \\
(n=110)\end{array}$ & Poor & 11 & 7 & 14 & 13 & $(0.02)$ & $\mathrm{NC} \vee \mathrm{NMH} \quad 0.324$ \\
\hline
\end{tabular}

*Inferential testing was not appropriate as cell occupancy was less than 5 in more than 20\% of cases (Pallant 2013)

** Level of significance set at 0.05

$* * *$ Reduced significance of alpha $=0.017(0.05 / 3)$ applied 
Table 4 Methods of learning and applying bioscience on placement (total number of responses for whole nursing sample and per field are presented)

\begin{tabular}{llllll}
\hline Survey question & Response options & $\begin{array}{l}\text { Total } \\
\text { nursing } \\
\text { sample }\end{array}$ & $\begin{array}{c}\text { Adult } \\
\text { field }\end{array}$ & $\begin{array}{c}\text { Child } \\
\text { field }\end{array}$ & $\begin{array}{l}\text { Mealth } \\
\text { field }\end{array}$ \\
\hline Can you tell us about the main & Discussions with your mentor & 89 & 33 & 29 & 27 \\
methods that you have used to & Patient care & 66 & 30 & 13 & 23 \\
apply your bioscience & Independent study & 52 & 26 & 16 & 10 \\
knowledge whilst on placement & Taught session & 42 & 20 & 13 & 9 \\
(tick all that apply): (n=110) & Personal reflection & 23 & 8 & 5 & 10 \\
& Online activities & 23 & 10 & 8 & 5 \\
& Other & 7 & 5 & 0 & 2 \\
\hline
\end{tabular}

